\title{
Thermal Correlators in LitTle String THEORY
}

\author{
Philip A. DeBoer* and Moshe Rozali ${ }^{\dagger}$ \\ Department of Physics and Astronomy \\ University of British Columbia
}

November 1, 2018

We calculate, using holographic duality, the thermal two-point function in finite temperature little string theory. The analysis of those correlators reveals possible instabilities of the thermal ensemble, as in previous discussions of the thermodynamics of little string theory. We comment on the dependence of the instability on the spatial volume of the system.

\section{Introduction and Summary}

Little string theories (LST) [1,2] are strongly interacting string theories, which exhibit phenomena quite different from their critical counterparts. Most notably, they do not contain a massless graviton in their spectrum. In their DLCQ definition [3] the strings appear in a very similar fashion to the critical strings, with the important difference being that the amplitude for splitting and joining strings is of order one, rather than being a free parameter that can be taken to be arbitrarily small.

It is clearly of interest to study these theories and compare their behavior to conventional field theories and to critical string theories. This allows for example an examination of nonlocal theories, without having the complications associated with gravity. Furthermore, this set of theories is very large, describing generic singular limits of string theory compactifications.

Studies of the thermodynamics of LST, using holographic duality [4], reveal qualitatively different features from more familiar theories $[5,6,7]$. The dual of LST at finite temperature is the CHGS cigar geometry [8]. In [5] it was pointed out that the infinite specific heat found in a tree level approximation of the dual geometry is an artifact of examining the latter in weak string coupling. The specific heat away from this limit was argued later to be negative $[6,9]$. For a nice review of these issues the reader may consult [10].

Negative specific heat is a generic feature of black holes in asymptotically flat spaces, so perhaps one should not be too surprised. However, the conjectured correspondence with a

*pdeboer@physics.ubc.ca

${ }^{\dagger}$ rozali@physics.ubc.ca 
well-defined boundary theory makes this feature somewhat mysterious. It would be useful to find a clear interpretation of the implied instability of the thermal ensemble. In [6] it was speculated that some mode, found earlier in the context of zero temperature "double scaled" LST [11], could become tachyonic at one loop level. Moreover, the analysis in [6] predicts an interesting dependence of the instability on the spatial volume of the system ${ }^{1}$.

However, the situation investigated in [11] is quite different - zero temperature theory far away on the Coulomb branch. One of the goals of the present paper is to formulate this conjecture, of being on the verge of instability, in the context of LST at finite temperature. Indeed we find that the system has modes which will destabilize it, given the correct mass shift at one loop. Furthermore we find complicated dependence of this phenomenon on the spatial momenta of the modes, which is reminiscent of the claim in [6].

Specifically we study two point functions in the the thermal state of LST. This is done using the holographic duals of these states, and utilizing the results of [12]. This calculation has no counterpart for a critical string theory, as Euclidean string theory on a thermal circle has no on-shell vertex operators. Indeed, the observables we concentrate on correspond to modes that are non-normalizable in a radial direction, and therefore are naturally interpreted as boundary rather than bulk properties. This is also the reason LST has off-shell correlators which can be calculated holographically, whereas critical string theory does not.

By and large, previous holographic probes of LST show patterns consistent with a free theory, apart from some puzzles (cutoff on off-shell momenta and some branch cuts in momentum space) which may signal a breakdown of perturbation theory [11]. Our correlators show no free theory patterns. There are no stable states, or even narrow resonances, revealed at that temperature. This is a sensible behavior of a strongly interacting system at a finite temperature.

There are also some puzzles, similar to the ones in [11], that suggest the importance of small corrections at large enough (Euclidean) frequencies. We comment on possible small modifications of the results that would make the amplitudes sensible in the high energy regime. In particular, there are some subtleties in the analytic continuation of the Euclidean results to real frequencies. Therefore, it would be useful to perform a real time calculation, in the gravity approximation, along the lines of [14]. We hope to return to that calculation in the future.

The organization of the paper is as follows. In section 2 we set up the holographic dual to LST at finite temperature, review the relevant world-sheet CFT, and construct the vertex operators. In section 3 we use that CFT to exhibit the thermal two-point function, using results from [12], and discuss its properties.

\footnotetext{
${ }^{1}$ Also there may be an interesting dependence on $k$, the number of 5-branes. However, here and in [6] we are working in leading order in the large $k$ limit.
} 


\section{Vertex Operators}

\subsection{Review of $S L(2, \mathbb{R})$ Results}

We begin by constructing the vertex operators of $S L(2, \mathbb{R}) / U(1)$ that survive a finite temperature GSO projection. For the application to LST, one needs only the non-normalizable modes in the holographic background, since these correspond to observables in the dual theory. For the vertex operators we concentrate on, such modes originate from the discrete representations of $S L(2, \mathbb{R})$ and their spectral flow images.

To begin, we need to set up the vertex operators of $S L(2, \mathbb{R})$. We use the notations of $[15,16]$. The $S L(2, \mathbb{R})$ level $k$ WZW action is

$$
\mathcal{S}=\frac{k}{8 \pi \alpha^{\prime}} \int_{\partial \Sigma} d^{2} \sigma \operatorname{Tr}\left(g^{-1} \partial g g^{-1} \partial g\right)+k \Gamma_{\mathrm{WZ}},
$$

where $g$ is an element of $S L(2, \mathbb{R})$ and $\Gamma_{\mathrm{WZ}}$ is the Wess-Zumino term

$$
\Gamma_{\mathrm{WZ}}=\frac{1}{24 \pi} \int_{\Sigma} d^{3} y \epsilon^{i j k} \operatorname{Tr}\left(g^{-1} \partial_{i} g g^{-1} \partial_{j} g g^{-1} \partial_{k} g\right) .
$$

The three-manifold $\Sigma$ has the world sheet as its boundary. Since $S L(2, \mathbb{R})$ has no non-trivial 3 -cycles, the level $k$ is not quantized ${ }^{2}$.

The conserved currents of this action are $J_{L, R}^{3, \pm}$. As a function of the left- and right-moving world sheet coordinates $z$ and $\bar{z}$ the currents are given by

$$
J_{L}^{3, \pm}(z)=k \operatorname{Tr}\left(\left(T^{3, \pm}\right)^{*} g^{-1} \partial g\right), \quad J_{R}^{3, \pm}(\bar{z})=k \operatorname{Tr}\left(T^{3, \pm} \bar{\partial} g g^{-1}\right)
$$

where $T^{3, \pm}$ are the generators of $S L(2, \mathbb{R})$. The currents can be expanded,

$$
J_{L}^{3, \pm}(z)=\sum_{n=-\infty}^{\infty} J_{n}^{3, \pm} e^{-i n z}, \quad J_{R}^{3, \pm}(\bar{z})=\sum_{n=-\infty}^{\infty} \bar{J}_{n}^{3, \pm} e^{-i n \bar{z}}
$$

The coefficients obey the Kac-Moody algebra:

$$
\begin{aligned}
{\left[J_{n}^{3}, J_{m}^{3}\right] } & =-\frac{k}{2} n \delta_{n,-m} \\
{\left[J_{n}^{3}, J_{m}^{ \pm}\right] } & = \pm J_{n+m}^{ \pm} \\
{\left[J_{n}^{+}, J_{m}^{-}\right] } & =-2 J_{n+m}^{3}+k n \delta_{n,-m} .
\end{aligned}
$$

The same algebra is satisfied by the modes of $\bar{J}$. The zero modes $J_{0}^{3, \pm}, \bar{J}_{0}^{3, \pm}$ obey the $S L(2, \mathbb{R})_{L} \times S L(2, \mathbb{R})_{R}$ algebra, appropriate for a particle moving on the $S L(2, \mathbb{R})$ group manifold.

\footnotetext{
${ }^{2}$ In the full geometry $k$ appears as the level of the WZW model on $\mathbb{S}^{3}$ and therefore is quantized.
} 
The left-moving Virasoro generators $L_{n}$ are,

$$
L_{0}=\frac{1}{k-2}\left[\frac{1}{2}\left(J_{0}^{+} J_{0}^{-}+J_{0}^{-} J_{0}^{+}\right)-J_{0}^{3} J_{0}^{3}+\sum_{m=1}^{\infty}\left(J_{-m}^{+} J_{m}^{-}+J_{-m}^{-} J_{m}^{+}-2 J_{-m}^{3} J_{m}^{3}\right)\right] \text {, }
$$

and for nonzero $n$

$$
L_{n}=\frac{1}{k-2} \sum_{m=1}^{\infty}\left(J_{n-m}^{+} J_{m}^{-}+J_{n-m}^{-} J_{m}^{+}-2 J_{n-m}^{3} J_{m}^{3}\right)
$$

likewise for the right-moving generators $\bar{L}_{n}$. These obey the Virasoro algebra with the central charge $c=\frac{3 k}{k-2}$.

The unflowed representations are generated, as usual, on top of representations of the zero mode algebra. We concentrate on the discrete representations - those are lowest (or highest) weight representations of $S L(2, \mathbb{R})$.

States in the discrete representations are described by two quantum numbers $j$ and $m$. The quadratic Casimir operator is given by $C_{2}(j)=\frac{1}{2}\left(J_{0}^{+} J_{0}^{-}+J_{0}^{-} J_{0}^{+}\right)-\left(J_{0}^{3}\right)^{2}=-j(j-1)$ and the eigenvalues of $J_{0}^{3}$ are given by $m$. The quantum number $m$ takes on the values $j+n$ where $n$ is any nonnegative integer. The state $|j ; m=j\rangle$ is annihilated by $J_{0}^{-}$as needed for a lowest-weight state. Unflowed representations of the full Kac-Moody algebra, based on these representations, are built in the usual way by the action of creation operators $J_{n}^{3, \pm}, n<0$. These representations are unitary, after imposing the Virasoro constraints, for $\frac{1}{2}<j<\frac{k-1}{2}$.

Additional representations of $S L(2, \mathbb{R})$ can be built using the operation of spectral flow. Unlike in the $S U(2)$ case, the spectral flow operation does not simply permute the representations, but rather gives new representations. This operation takes $J_{n}^{3, \pm} \rightarrow J_{n}^{3, \pm ; w}$ given by

$$
J_{n}^{3 ; w}=J_{n}^{3}-\frac{k w}{2} \delta_{n, 0}, \quad J_{n}^{+; w}=J_{n+w}^{+}, \quad J_{n}^{-; w}=J_{n-w}^{-} .
$$

The integer $w$ parameterizes the amount of spectral flow ${ }^{3}$.

Under spectral flow the Virasoro generators change as

$$
L_{n}^{w}=L_{n}+w J_{n}^{3}-\frac{k w^{2}}{4} \delta_{n, 0}
$$

and similarly for the $\bar{L}_{n}$.

The eigenvalues of $\left(J_{0}^{w}\right)^{2}, J_{0}^{3 ; w}$ are denoted by $-J(J-1)$ and $M$ respectively. To maintain unitarity, after imposing the Virasoro constraints, the eigenvalue $J$ of the flowed quadratic Casimir must satisfy $\frac{1}{2}<J<\frac{k-1}{2}$.

We will concentrate on vertex operators which are tachyons in the $S L(2, \mathbb{R}) / U(1)$ part of the geometry, namely states which are created only by the zero modes of the $S L(2, \mathbb{R})$

\footnotetext{
${ }^{3}$ We consider here flow which acts the same way for both left and right movers. The flow which acts with opposite signs will create winding around the time-like direction of $S L(2, \mathbb{R})$, when it is compact [17].
} 
currents (with possible oscillator modes from other parts of the CFT). The vertex operators of such states, in flowed discrete representations of $S L(2, \mathbb{R})$ with eigenvalues $M$, $\bar{M}$, will be denoted $\Phi_{J M \bar{M}}^{w}$.

\section{$2.2 \quad$ Restriction to $S L(2, \mathbb{R}) / U(1)$}

To obtain the Euclidean cigar geometry the diagonal $U(1)$ subgroup of the $S L(2, \mathbb{R})_{L} \times$ $S L(2, \mathbb{R})_{R}$ symmetry should be modded out. This $U(1)$ is generated by $J=\left(J_{L}^{3}+\bar{J}_{R}^{3}\right)$. The gauging is described in detail in [16], and we follow their notation.

After introducing a gauge field and bosonizing, the action involves a new compact scalar field, with the wrong-sign kinetic term,

$$
\mathcal{S}_{\phi}=-(k / 4 \pi) \int d^{2} z \partial \phi \bar{\partial} \phi
$$

The original gauge symmetry is manifested by the requirement that all physical operators must commute with the BRST charge

$$
Q=\oint d z c\left(J_{L}^{3}+\frac{i}{2} k \partial \phi\right)+\text { complex conjugate. }
$$

The simplest vertex operators in the $S L(2, \mathbb{R}) / U(1)$ are of the form

$$
V=e^{i q_{L} \phi_{L}(z)+i q_{R} \phi_{R}(\bar{z})} \Phi_{J M \bar{M}}^{w}
$$

The BRST invariance then sets $q_{L}=-m, q_{R}=\bar{m}$. Note it is the eigenvalues of the unflowed generators $J_{0}^{3}, \bar{J}_{0}^{3}$ which appear here.

The compactification of $\phi$ further implies that $\frac{q_{L}-q_{R}}{k}$ is an integer $n$, identified in [16] as the winding around the asymptotic circle of the cigar. This translates to the restriction $\frac{M+\bar{M}}{k}$ is an integer $n-w$. We will also see that correlation functions depend only on this combination, and not on $n$ and $w$ separately. We conclude therefore that without loss of generality one can start with the unflowed representations of $S L(2, \mathbb{R})$ - the winding will be generated using the compact scalar $\phi^{4}$.

The vertex operator has conformal weights (with respect to the unflowed $L_{0}, \overline{L_{0}}$ ),

$$
\begin{aligned}
& h_{J p n}=-\frac{J(J-1)}{k-2}+\frac{(p+n k)^{2}}{4 k}, \\
& \bar{h}_{J p n}=-\frac{J(J-1)}{k-2}+\frac{(p-n k)^{2}}{4 k},
\end{aligned}
$$

where we denote $p=m-\bar{m}$ to be the momentum around the asymptotic circle. This is the spectrum found in [16]. Note that it is the Casimir operator with restricted domain which

\footnotetext{
${ }^{4}$ This construction of vertex operators of $S L(2, \mathbb{R})$, was sketched in [18], using the language of parafermions, where the last fact is more transparent.
} 
appears in the mass formulas,

$$
\begin{aligned}
\Delta=L_{0}+\overline{L_{0}}-1 & =-\frac{J(J-1)}{k-2}+\frac{p^{2}}{2 k}+\frac{k n^{2}}{2} \\
L_{0}-\overline{L_{0}} & =p n .
\end{aligned}
$$

This justifies the interpretation of $p, n$ as momentum and winding, respectively, around the Euclidean time direction.

We are interested in exploring correlators as a function of the Euclidean momentum $p$. It is therefore essential not to restrict the possible values of $p$. For that reason we choose $n=0$; otherwise the level matching condition will make it difficult to construct a series of allowed vertex operators with the different momenta, to be interpreted as Fourier modes of a single object $^{5}$. It may be expected that the set of observables does not change at finite temperature, and the quantum number $n$ has no clear zero temperature limit in the boundary theory.

However, we will see that the set of observables with $n=0$ does not allow exploration of all possible momenta (for finite $k$ ). This is essentially due to the same bound on the allowed off-shell momenta found in [11]. In the present context, this puzzle may eventually be resolved by making use of observables with $n \neq 0$. We comment further on this point below.

We therefore choose to work with graviton vertex operators, which in addition to the wave function in the $S L(2, \mathbb{R}) / U(1)$ part have oscillator excitations in the left and right moving sectors of the free $\mathrm{CFT}$ on $\mathbb{R}^{5}$. The $\mathbb{S}^{3}$ part remains in its ground state.

\subsection{Worldsheet Supersymmetry}

To supersymmetrize the theory, eight Majorana-Weyl fermions are added to each sector of the model. These fermions are free after a suitable chiral rotation.

The addition of fermions changes the scaling dimension of vertex operators. Since the

zero point energy on the world-sheet is altered, the term $\frac{2 J(J-1)}{k(k-2)}$ is added to both $h_{J p n}$ and $\bar{h}_{J p n}$ so that this scaling becomes

$$
h_{J p n}=-\frac{J(J-1)}{k}+\frac{(p+n k)^{2}}{4 k}
$$

and likewise for $\bar{h}_{J p n}$.

The GSO projection used here breaks spacetime supersymmetry because it imposes different periodicity conditions on spacetime bosons and fermions around the compact Euclidean time direction. Spacetime fermions are anti-periodic while bosons are periodic.

Requiring spacetime fermions to be anti-periodic, together with modular invariance, introduces new phase factors into the sum over spin structures. This was done by Atick and

\footnotetext{
${ }^{5}$ Such vertex operators will have to involve oscillator excitations which are left-right asymmetric, and generally carry spacetime quantum numbers.
} 
Witten [19] using the functions $U_{i}(p, n)$, which modify the usual phases of Type II strings at zero temperature. Explicitly ${ }^{6}$, these four functions are

$$
\begin{aligned}
U_{1}(p, n) & =\frac{1}{2}\left(-1+(-1)^{p}+(-1)^{n}+(-1)^{p+n}\right), \\
U_{2}(p, n) & =\frac{1}{2}\left(1-(-1)^{p}+(-1)^{n}+(-1)^{p+n}\right), \\
U_{3}(p, n) & =\frac{1}{2}\left(1+(-1)^{p}+(-1)^{n}-(-1)^{p+n}\right), \\
U_{4}(p, n) & =\frac{1}{2}\left(1+(-1)^{p}-(-1)^{n}+(-1)^{p+n}\right) .
\end{aligned}
$$

Using the definition $U_{i}=U_{i}(p, n)$ for states with momentum $p$ and winding $n$ the desired one loop partition function is given by the modular invariant trace

$$
\begin{aligned}
\operatorname{Tr}\left(e^{i \tau \mathcal{H}}\right. & {\left[U_{3}-(-1)^{F_{\tau}} U_{4}-(-1)^{F_{\sigma}} U_{2}-(-1)^{F_{\sigma}+F_{\tau}} U_{1}\right] } \\
& {\left.\left[U_{3}-(-1)^{\tilde{F}_{\tau}} U_{4}-(-1)^{\tilde{F}_{\sigma}} U_{2} \pm(-1)^{\tilde{F}_{\sigma}+\tilde{F}_{\tau}} U_{1}\right]\right), }
\end{aligned}
$$

where $F_{\sigma, \tau}$ are the left-moving fermion number operators acting in the $\sigma, \tau$ directions and $\mathcal{H}=L_{0}+\overline{L_{0}}$ is the Hamiltonian. The signs between the terms have been chosen to correspond to the Type IIA,B string.

The vertex operators we are interested in survive the zero temperature GSO projection. The analysis in the previous section confirms that all momentum modes of them survive, enabling us to discuss the two point function as an analytic function of frequency.

\subsection{Mass-Shell Conditions}

Each one of our vertex operator carries quantum numbers corresponding to its dependence on various coordinates. One of the general properties of holography is the relation between on-shell bulk quantities and off-shell boundary correlators. For this to be possible, it is essential that the observables have a sufficient range of bulk quantum numbers which are invisible from the boundary perspective. One should be able to solve the bulk mass-shell conditions for each value of the boundary quantum numbers. In our case the latter include the spatial momenta $q_{i}$, and the Matsubara frequencies $p$. Even in the zero temperature analysis of [11] this proves to be impossible, and we will see similar issues arise here.

The graviton vertex operator in the full target space is given by

$$
\left.g_{i j}\left(q_{i}, p\right)=e^{-\phi-\bar{\phi}} \psi_{(i} \bar{\psi}_{j}\right) e^{i q_{i} X^{i}} \Phi_{J p}
$$

where $\phi, \bar{\phi}$ are the bosonized superghost fields and $X^{i}, \psi_{i}, \bar{\psi}_{i}$ are the free bosons and fermions in the $\mathbb{R}^{5}$ component of the full CFT. The indices $i, j$ in this expression are symmetrized.

\footnotetext{
${ }^{6} \mathrm{We}$ are using here the flat space result since we will be mainly interested in the leading results in the large $k$ limit, and in the limit $k \rightarrow \infty$ one recovers a flat space.
} 
The vertex operator $\Phi_{J p}$ is the $S L(2, \mathbb{R}) / U(1)$ vertex operator discussed above, with the winding set to zero.

In writing $g_{i j}\left(q_{i}, p\right)$ as a function of $q_{i}, p$ only we imply that other quantum numbers are to be regarded as implicit function of those, given by the mass-shell condition. The massshell condition arises as the requirement that $g_{i j}$ be a conformally invariant vertex operator. Specifically,

$$
\frac{q^{2}}{2}-\frac{J(J-1)}{k}+\frac{p^{2}}{4 k}=0
$$

so that $J$ is a function of the off-shell quantum numbers $q_{i}, p$. In particular, the bound on $J$ translates here to a bound on those quantum numbers, exactly as in [11].

\section{Correlation Functions}

We are now ready to exhibit the thermal two-point functions of the operators we are interested in. The ingredients of this calculation were performed in [12], and reviewed in $[11,18]$.

In the notation of [18] the two-point correlator of functions (tachyon vertex operators) on $S L(2, \mathbb{C}) / U(1)$, the Euclidean version of $S L(2, \mathbb{R})$, can be calculated in the $x$-space (defined below) as

$$
\left\langle\Phi_{J}\left(x_{1}\right) \Phi_{J}^{\prime}\left(x_{2}\right)\right\rangle=\frac{\delta\left(J-J^{\prime}\right) B(J)}{\left|z_{12}\right|^{2 h}\left|\bar{z}_{12}\right|^{2 \bar{h}}\left|x_{12}\right|^{4 J}},
$$

where $h, \bar{h}$ are the conformal weights, and $x_{12}=x_{1}-x_{2}$. The $x$ basis is related to the $M$ basis, used in the present paper, via

$$
\Phi_{J M \bar{M}}=\int \frac{d^{2} x}{|x|^{2}} x^{J-M} x^{J-\bar{M}} \Phi_{J}(x) .
$$

This gives [18]

$$
\left\langle\Phi_{J M \bar{M}} \Phi_{J^{\prime} M^{\prime} \bar{M}^{\prime}}\right\rangle=\frac{\pi \delta^{2}\left(M+M^{\prime}\right) \delta(J-J) B(J)}{\left|z_{12}\right|^{2 h}\left|\bar{z}_{12}\right|^{2 \bar{h}} \gamma(2 J)} \frac{\Gamma(J+M) \Gamma(J-\bar{M})}{\Gamma(1-J+M) \Gamma(1-J-\bar{M})} .
$$

The functions $B(J)$ and $\gamma(2 J)$ are defined in $[12,11]$ :

$$
B(J)=\frac{k \nu^{1-2 J}}{\pi \gamma\left(\frac{2 J-1}{k}\right)}
$$

and

$$
\gamma(x)=\frac{\Gamma(x)}{\Gamma(1-x)} .
$$

The constant $\nu$ and the function $B(J)$ both equal 1 in the large $k$ limit we are taking.

In calculating the spacetime two point function, one has to divide by the volume of the conformal group. This normally makes the two point function vanish, but in this context it 
is compensated by the volume of target space $[20,6,18]$. This leaves a finite piece, which was calculated in [18].

In making the correlation function of our vertex operators $g_{i j}$, the additional ingredients will make little difference. They will modify the conformal weights in the denominator of (21), and will multiply the correlator by a polarization dependent tensor in the flat spatial directions.

The two point function of the vertex operators $g_{i j}\left(q_{i}, p\right)$ is then given by:

$$
\begin{array}{r}
\left\langle g_{i j}\left(q_{i}, p\right) g_{k l}\left(q_{i}^{\prime}, p^{\prime}\right)\right\rangle \propto\left(\delta_{i k} \delta_{j l}+\delta_{i k} \delta_{j l}\right) \delta^{5}\left(q+q^{\prime}\right) \delta\left(p+p^{\prime}\right) \times \\
\quad \times \frac{1}{2 J-1} \frac{1}{\gamma(2 J)} \frac{\Gamma(J+M) \Gamma(J-\bar{M})}{\Gamma(1-J+M) \Gamma(1-J-\bar{M})}
\end{array}
$$

Here $M, \bar{M}$ and $J$ are regarded as functions of the quantum numbers $q_{i}, p$. We now proceed to comment on the structure of this result and its analytic continuation to real frequencies.

\subsection{Euclidean Space}

The temperature Green's function can be written as a function of the Matsubara frequencies $p$ as

$$
\mathcal{G} \sim \frac{1}{\gamma\left(1+\sqrt{1+2 k q^{2}+p^{2}}\right)} \frac{\Gamma^{2}\left(\frac{1}{2}+\frac{1}{2} \sqrt{1+2 k q^{2}+p^{2}}+\frac{p}{2}\right)}{\Gamma^{2}\left(\frac{1}{2}-\frac{1}{2} \sqrt{1+2 k q^{2}+p^{2}}+\frac{p}{2}\right)} .
$$

This is found using the conditions $M=p / 2, \bar{M}=-p / 2$, and the solution

$$
J=\frac{1}{2}+\frac{1}{2} \sqrt{1+2 k q^{2}+p^{2}}
$$

to the mass-shell condition (18).

A second solution to the mass-shell condition is discarded as it violates the lower bound on $J$. The upper bound imposes a constraint on a combination of the Euclidean energy $p$ and the transverse momentum $q$,

$$
\sqrt{1+2 k q^{2}+p^{2}}<k-2 \text {. }
$$

There is an additional condition $M=J-n=p / 2$, for $n$ an integer of definite sign, since those are the allowed values in the discrete representations. Positive $n$ corresponds to a highest-weight representation, while negative $n$ corresponds to a lowest-weight representation. This translates, using the mass-shell condition, to

$$
\frac{k q^{2}}{2}+n^{2}=J(2 n-1)
$$

Since $J$ is positive it is clear that $n$ must be positive as well, so only the highest-weight representations are allowed. 
So we see that only a discrete set of points in the frequency-momentum space is obtained by the Euclidean string theory calculation. Our approach is to analytically continue the result as needed, and attempt interpretation of the correlator as a function of Lorentzian frequencies ${ }^{7}$.

\subsection{Minkowski Space}

The temperature Green's function $\mathcal{G}(p)$ can be analytically continued to the retarded Green's function $G_{R}(\omega)$ using $p \rightarrow-i \omega+\delta$. The analytic continuation of $\mathcal{G}$ is not unique since it can, for example, be multiplied by a phase which vanishes at the poles. Typically one requires a fall-off condition for large Euclidean frequencies in order to fix the analytic continuation uniquely.

These fall-off conditions require knowledge of the correlators at arbitrarily high Euclidean frequencies. As our frequencies are a priori bounded (for any finite $k$ ), we are unable to discuss those fall-off conditions. Even without modifying the correlator found by Teschner (which is exact in tree level string theory), it is possible that small modifications to the mass shell condition (18) will result in satisfying the asymptotic fall-off conditions. We assume this to be the case; as mentioned in the introduction a real time calculation of the retarded Green's function will be useful to confirm or refute this point.

We assume therefore that the form of the correlator (25) applies to Lorentzian frequencies as well. The retarded Green's function is therefore taken to be

$$
G_{R} \sim \frac{\Gamma\left(-\sqrt{1+2 k q^{2}-\omega^{2}}\right) \Gamma^{2}\left(\frac{1}{2}+\frac{1}{2} \sqrt{1+2 k q^{2}-\omega^{2}}+\frac{i \omega}{2}\right)}{\Gamma\left(1+\sqrt{1+2 k q^{2}-\omega^{2}}\right) \Gamma^{2}\left(\frac{1}{2}-\frac{1}{2} \sqrt{1+2 k q^{2}-\omega^{2}}+\frac{i \omega}{2}\right)} .
$$

In a retarded Green's function an instability will manifest itself as a pole (or other type of singularity, for more complex instabilities) below the real axis. This will be a process which grows, rather than decays, as a function of time. As we are looking for modes which may destabilize the system upon including arbitrarily small corrections, the interesting structure for our purposes occurs on the real axis. This structure represents modes that are stable in the thermal ensemble, an unusual feature.

The retarded Green's function found above has only one set of singularities, coming from the factor $\Gamma\left(-\sqrt{1+2 k q^{2}-\omega^{2}}\right)$. The singularities are located at

$$
\omega= \pm \sqrt{1+2 k q^{2}-n^{2}}
$$

where $n$ is a nonnegative integer. These points are located along the real and imaginary axes. The points along the real axis are bounded (for generic ${ }^{8}$ spatial momenta $q_{i}$ ),

$$
|\omega|<\sqrt{1+2 k q^{2}}
$$

\footnotetext{
${ }^{7}$ We note that the original derivation of the correlators [12] utilizes analytic continuation as well. It would be interesting to explore alternative Lorentzian continuations, along the lines of [13].

${ }^{8}$ There are some more possibilities at special kinematic points, but as those will be sensitive to small corrections, we concentrate on generic kinematics.
} 
We see that a large number of possible instabilities is present for non-zero spatial momenta $q^{2}$, a number of order $k q^{2}$. We note that the holographic dual to LST contains a continuum of modes above a gap, whose role in the theory is unclear. A typical momentum of those modes, just above the gap, is $q^{2} \sim \frac{1}{k}$. Therefore there are order $k$ possible "nearly destabilizing" modes, and nearly all of them are above the gap. The frequencies of those modes are much smaller than $k$ (where our analysis is expected to receive corrections).

On the other hand, for zero spatial momentum there is only one such possible mode, at $|\omega|=1^{9}$. We see a similar picture to the one suggested in [6], where the instability depends on the spatial volume (or the spatial momentum in our case).

The retarded Green's function also has a set of zeros. These are on the imaginary axis, so in particular they can eliminate a possible divergence only for special values of $q_{i}$, the spatial momentum.

\section{Acknowledgments}

We are thankful for useful conversations with Micha Berkooz, Mark Laidlaw, Gordon Semenoff and Bill Unruh. The work was supported in part by NSERC.

\section{References}

[1] M. Berkooz, M. Rozali and N. Seiberg, "Matrix Description of M-theory on $T^{4}$ and $T^{5}$," Phys. Lett. B 408, 105 (1997) [arXiv:hep-th/9704089].

[2] N. Seiberg, "New theories in six dimensions and matrix description of M-theory on T**5 and T**5/Z(2)," Phys. Lett. B 408, 98 (1997) [arXiv:hep-th/9705221].

[3] O. Aharony, M. Berkooz, S. Kachru, N. Seiberg and E. Silverstein, "Matrix description of interacting theories in six dimensions," Adv. Theor. Math. Phys. 1, 148 (1998) [arXiv:hep-th/9707079];

E. Witten, "On the conformal field theory of the Higgs branch," JHEP 9707, 003 (1997) [arXiv:hep-th/9707093].

[4] O. Aharony, M. Berkooz, D. Kutasov and N. Seiberg, "Linear dilatons, NS5-branes and holography," JHEP 9810, 004 (1998) [arXiv:hep-th/9808149].

[5] T. Harmark and N. A. Obers, "Hagedorn behaviour of little string theory from string corrections to NS5-branes," Phys. Lett. B 485, 285 (2000) [arXiv:hep-th/0005021].

M. Berkooz and M. Rozali, "Near Hagedorn dynamics of NS fivebranes, or a new universality class of coiled strings," JHEP 0005, 040 (2000) [arXiv:hep-th/0005047].

\footnotetext{
${ }^{9} \mathrm{An}$ additional potential pole at $\omega=0$ is eliminated by a zero at that frequency.
} 
[6] D. Kutasov and D. A. Sahakyan, "Comments on the thermodynamics of little string theory," JHEP 0102, 021 (2001) [arXiv:hep-th/0012258].

[7] K. Narayan and M. Rangamani, "Hot little string correlators: A view from supergravity," JHEP 0108, 054 (2001) [arXiv:hep-th/0107111].

A. Buchel, "On the thermodynamic instability of LST," [arXiv:hep-th/0107102].

M. Rangamani, "Little string thermodynamics," JHEP 0106, 042 (2001) [arXiv:hepth/0104125].

T. Harmark and N. A. Obers, "Hagedorn behavior of little string theories," [arXiv:hepth/0010169].

[8] C. G. Callan, S. B. Giddings, J. A. Harvey and A. Strominger, "Evanescent Black Holes," Phys. Rev. D 45, 1005 (1992) [arXiv:hep-th/9111056].

E. Witten, "On string theory and black holes," Phys. Rev. D 44, 314 (1991).

[9] V. Kazakov, I. K. Kostov and D. Kutasov, "A matrix model for the two-dimensional black hole," Nucl. Phys. B 622, 141 (2002) [arXiv:hep-th/0101011].

[10] D. Kutasov, "Introduction to little string theory," Prepared for ICTP Spring School on Superstrings and Related Matters, Trieste, Italy, 2-10 Apr 2001.

[11] A. Giveon and D. Kutasov, "Little string theory in a double scaling limit," JHEP 9910, 034 (1999) [arXiv:hep-th/9909110].

A. Giveon and D. Kutasov, "Comments on double scaled little string theory," JHEP 0001, 023 (2000) [arXiv:hep-th/9911039].

[12] J. Teschner, "On structure constants and fusion rules in the SL(2,C)/SU(2) WZNW model," Nucl. Phys. B 546, 390 (1999) [arXiv:hep-th/9712256].

J. Teschner, "The mini-superspace limit of the SL(2,C)/SU(2) WZNW model," Nucl. Phys. B 546, 369 (1999) [arXiv:hep-th/9712258];

J. Teschner, "Operator product expansion and factorization in the H-3+ WZNW model," Nucl. Phys. B 571, 555 (2000) [arXiv:hep-th/9906215].

[13] P. Kraus, H. Ooguri and S. Shenker, "Inside the horizon with AdS/CFT," [arXiv:hepth/0212277].

[14] C. P. Herzog and D. T. Son, "Schwinger-Keldysh propagators from AdS/CFT correspondence," [arXiv:hep-th/0212072];

G. Policastro, D. T. Son and A. O. Starinets, "From AdS/CFT correspondence to hydrodynamics," JHEP 0209, 043 (2002) [arXiv:hep-th/0205052].

G. Policastro, D. T. Son and A. O. Starinets, "From AdS/CFT correspondence to hydrodynamics. II: Sound waves," [arXiv:hep-th/0210220]. 
C. P. Herzog, "The hydrodynamics of M-theory," JHEP 0212, 026 (2002) [arXiv:hepth/0210126].

D. T. Son and A. O. Starinets, "Minkowski-space correlators in AdS/CFT correspondence: Recipe and applications," JHEP 0209, 042 (2002) [arXiv:hep-th/0205051].

[15] J. M. Maldacena and H. Ooguri, "Strings in $\operatorname{AdS}(3)$ and SL(2,R) WZW model. I," J. Math. Phys. 42, 2929 (2001) [arXiv:hep-th/0001053].

J. M. Maldacena, H. Ooguri and J. Son, "Strings in $\operatorname{AdS}(3)$ and the $\operatorname{SL}(2, R)$ WZW model. II: Euclidean black hole," J. Math. Phys. 42, 2961 (2001) [arXiv:hepth/0005183].

[16] R. Dijkgraaf, H. Verlinde and E. Verlinde, "String propagation in a black hole geometry," Nucl. Phys. B 371, 269 (1992).

[17] A. Rajaraman and M. Rozali, "Boundary states for D-branes in AdS(3)," Phys. Rev. D 66, 026006 (2002) [arXiv:hep-th/0108001].

[18] J. M. Maldacena and H. Ooguri, "Strings in $\operatorname{AdS}(3)$ and the SL(2,R) WZW model. III: Correlation functions," Phys. Rev. D 65, 106006 (2002) [arXiv:hep-th/0111180].

[19] J. J. Atick and E. Witten, "The Hagedorn Transition And The Number Of Degrees Of Freedom Of String Theory," Nucl. Phys. B 310, 291 (1988).

[20] N. Seiberg, "Notes On Quantum Liouville Theory And Quantum Gravity," Prog. Theor. Phys. Suppl. 102, 319 (1990). 